\title{
Association of HMGB1, S100A12 and IL-17A Expression with IVIG-Resistant Kawasaki Disease and Treatment Options
}

\section{Deying Liu}

Wuhan Women and Children Medical Care Center

Pan Liu

United Hospital

Fan Liu

Wuhan Women and Children Medical Care Center

Wei Yin

Wuhan Women and Children Medical Care Center

Yan Ding ( $\nabla$ dingyanmx@163.com )

Wuhan Women and Children Medical Care Center

Research article

Keywords: Kawasaki disease 1, IVIG-resistant 2, HMGB1 3, S100A12 4, IL-17A 5

Posted Date: August 18th, 2020

DOI: https://doi.org/10.21203/rs.3.rs-51161/v1

License: (c) (1) This work is licensed under a Creative Commons Attribution 4.0 International License. Read Full License 


\section{Abstract}

Background: Kawasaki disease (KD) is a medium vessel vasculitis of unknown aetiology that predominantly affecting coronary arteries. The damage-associated molecular pattern molecules (DAMPs) such as HMGB1, S100A12 and IL-17A have been reported to predict poor response to IVIG. Here, we explored the the role of HMGB1, S100A12 and IL-17A in the detection of intravenous immunoglobulin (IVIG)-resistant in KD patients, and to investigate the value of different adjunctive therapy.

Method: $126 \mathrm{KD}$ patients and as well as age- and sex-matched 16 febrile control subjects were enrolled in our study. The fresh peripheral blood were collected from KD patients and febrile controls, analyzed the demographic or clinical data and various laboratory parameters. We also measured changes in serum levels of IL-17A and mRNA expression levels of HMGB1 and S100A12 were tested in IVIG-resistant KD patients. Further we explored the association between coronary arteries lesions and different treatment options about IVIG retreatment, methylprednisolone and infliximab for IVIG-resistant KD patients.

Result: Regarding laboratory parameters, KD individuals were found to have lower levels of lymphocyte $(\mathrm{L}) \%$, prealbumin, $\mathrm{CD} 4^{+}, \mathrm{CD} 8^{+}$and higher levels of $\mathrm{WBC}$, neutrophil $(\mathrm{N}) \%$, CRP, ESR, NT-proBNP, $A L T, C D 4^{+} / \mathrm{CD}^{+}(\mathrm{P}<0.05$ or $\mathrm{P}<0.01)$. For KD group, the $53 \mathrm{IVIG}$-resistant patients had significantly higher levels of S100A12, HMGB1, serum IL-17A, N\%, CRP, NT-proBNP, TBIL, ALT, AST and lower levels of L\%, PLT $(P<0.05$ or $P<0.01)$ in comparison to the IVIG-responsive patients. For patients with IVIG-resistant, IVIG retreatment, methylprednisolone or infliximab were used. Methylprednisolone showed better in improving clinical symptoms and CRP than the IVIG retreatment and infliximab $(P>0.05)$.

Conclusion: IVIG-resistant was associated with overreaction of inflammation. The levels of HMGB1,S100A12 and IL-17A suggested to be reliable predictors for IVIG-resistant in KD. In addition, the adjunctive therapy of methylprednisolone and infliximab showed more effective in relieving clinical symptoms than IVIG retreatment.

\section{Introduction}

Kawasaki disease (KD)is an acute febrile illness that causes systemic vasculitis in young children[1]. KD is now the most common cause of acquired heart disease in developing countries[2]which can lead to serious coronary artery lesion[3, 4]. Timely a single infusion of $2 \mathrm{~g} / \mathrm{kg}$ intravenous immunoglobulin (IVIG) along with aspirin [5] can declined the incidence of coronary artery lesions (CALs) effectively[1, 6]. However, $10-20 \%$ of KD patients develop recurrent or persistent fever after first dose IVIG treatment are classified as IVIG resistant[7, 8]. These IVIG resistant patients have a higher risk of CALs compared with responders. Therefore, identifying some biomarkers of patients who are likely to be IVIG-resistant would guide the choice of treatment option. So as to prevent CALs by inhibiting the inflammation reaction in the early stage.

The DAMPs such as $\mathrm{S} 100$ calcium-binding protein A12 (S100A12) and high-mobility group protein B1 (HMGB1) have been reported to be a sensitive indicator for disease activity and inflammation in many 
inflammatory disorders. Studies have found HMGB1 and S100A12 are closely associated with the development of CAL in $\operatorname{KD}[5,9,10]$. These markers are released from stressed cells and binding with receptor for advanced glycation end products (RAGE) or Toll-like receptors (TLRs) to activate the NF-KB pathway to produce endothelial damage and lead to CALs development in KD patients[11]. In addition, Elevated Th17 cells and decreased T regulatory cells have also been demonstrated in the acute stage of KD. Plasma levels of Th17 and Interleukin-17A (IL-17A) are also highly expressed in KD. IL-17A, tumor necrosis factora (TNF-a) and IL- 6 secreted by Th17 cells have proinflammatory properties, which can collectively mobilize, recruit and activate neutrophils, thus mediating inflammation of the tissues[12-14].

IVIG resistance is a high risk factor for coronary involvement[15]. Here we aim to identify the clinical features and laboratory factors that are predictive of IVIG resistant KD. And early pre-judgment of nonresponse to IVIG has an important guiding role in managing KD[16]. Higher level of C-reactive protein (CRP), neutrophils(N), AST, NT-proBNP and lower level of platelet(PLT) counts have been known as the risk factors for IVIG non-response KD[17-22]. Thus, IVIG-resistant patients are associated with severe inflammatory reactions, adjunctive treatment are needed to interfere the overwhelming inflammatory process[23]. To identify predictive biomarkers which related to IVIG resistance in the early stage is meaningful. In this study, we measured some laboratory characteristics as potential risk factors for IVIG resistance KD. And we found HMGB1, S100A12 and IL-17A in children KD are at high risk for IVIG resistance.

Recently, although several adjunctive therapies such as IVIG retreatment, corticosteroids, infliximab and Others are available, comparative robust data on which to IVIG-resistant treatment decisions are scarce[24]. IVIG appears to have a wide range of anti-inflammatory effect, and possible mechanisms of action include regulation of cytokine production, increased regulation of T-cell activity, neutralization of toxins and so on $[1,25]$. Methylprednisolone attributes to suppression of persistent vascular inflammation. Infliximab (TNF-a blockade) binds specifically to human TNF- $a$ and is indicated for the treatment of immune-modulated inflammatory disorders. Thus, We summarized the experience with three different IVIG retreatment options to guide the choice of Optimal therapeutic agents to clinicians for the children with IVIG resistance.

\section{Methods}

\section{Patients}

Written informed consents were obtained from parents or guardians of all study participants. $126 \mathrm{KD}$ patients who were diagnosed according to the criteria established by the American Heart Association(AHA) in Wuhan Children's Hospital from 2015 to 2019[1]. The diagnosis of classic KD is according to the presence of fever at least 5 days and the presence of $\geq 4$ of the 5 following principal clinical criteria: 1 . strawberry tongue, fissure and erythema lips $₫ 2$. bilateral nonpurulent conjunctivitis; 3 . maculopapular orerythema multiforme-like rashes; 4. redness and swelling of the hands and feet or periungual membrane desquamation; 5 . unilateral cervical lymphadenopathy. We took the peripheral 
blood samples from all patients when they were admitted to hospital in acute stage. Another 16 patients with an acute febrile infectious disease were selected as a normal control group. Moreover, KD group was further divided into IVIG-responsive group and IVIG-resistant group.

All the KD patients were treated with an initial IVIG infusion at $2 \mathrm{~g} / \mathrm{kg}[26]$. We defined IVIG-responsive as defervescence within 36 to 48 hours after the initial IVIG infusion and no recurrence(temperature $>38^{\circ} \mathrm{C}$ ), IVIG-resistant patients were persistent fever $\left(T>38^{\circ} \mathrm{C}\right)$ beyond 36 to $48 \mathrm{~h}$ after standard therapy[5, 26]. In our study, there were $53 \mathrm{KD}$ patients turn into IVIG-resistant after initial IVIG and Acetylsalicylic Acid (ASA) therapy. Among them, 23 IVIG-resistance patients were continued with second round IVIG at $2 \mathrm{~g} / \mathrm{kg}$; 26 IVIG-resistant patients received methylprednisolone $(20 \mathrm{mg} / \mathrm{kg} / \mathrm{d}$ for 3 consecutive days); and 4 patients were treated with infliximab ( $5 \mathrm{mg} / \mathrm{kg}$ intravenously over 2 hours). Laboratory indicators at admission(KD1), after innitial IVIG(KD2), and at different treatment(KD3) laboratory variables were recorded. All of them were followed up regularly in the outpatient department.

\section{Blood laboratory collection}

All participants underwent a routine blood test, CRP, procalcitonin (PCT), erythrocyte sedimentation rate (ESR), serum albumin(ALB), total bilirubin(TBIL), AST, ALT, NT-proBNP, CD $4^{+}$and CD $8^{+}$T cells (\%) etc. All $\mathrm{KD}$ patients laboratory data were obtained at admission before IVIG therapy.

\section{Quantitative RT-PCR}

The expression levels of mRNA for S100A12 and HMGB1 in whole blood were measured in 73 IVIGresponsive and 53 IVIG-resistant KD patients using real-time PCR. Total RNA from whole blood of KD patients was isolated by using RNA-Trizol (Takara, Dalian, China), and reverse transcription (RNA $\rightarrow$ CDNA) was carried out following the the manufacturer's instruction of kit (PrimeScript ${ }^{\mathrm{TM}}$ RT Master Mix kit, Code: RR036A). After the template cDNA was synthesized, the amplification steps and the reaction conditions were followed with the instruction of kit ( SYBR® Premix Ex Taq ${ }^{\mathrm{TM}}$ kit (Catalogue No: RR420A). The procedure were repeated for three times and the data were analyzed using the $2^{-\Delta \Delta C t}$ method[27]. Sequences of primers for real-time PCR are included in Table 1.

\section{Serum IL-17A Levels}

We measured the level of serum IL-17A in patients by the ELISA in according to the manufacturer's instruction[27]. The sensitivity of the human IL-17A ELISA kit (Elabscience, Catalogue No: E-EL-H0105c) was $18.75 \mathrm{pg} / \mathrm{mL}$, and the intra- and inter-assay precision was below $10 \%$.

\section{Statistical Analysis}

Data are expressed as the mean \pm SD for a percentage for categorical variables. We analyzed demographic, quantitative data or mRNA expression levels with Student's t-test or one-way ANOVA. SPSS version 12.0 software was used for statistical analyses. The figures in this study were generated by using GraphPad Prism 5.0 software. 


\section{Results}

\section{Laboratory characteristics of the KD group and control group}

All laboratory data used in this study were obtained at admission before the initiation of therapy. The variance of laboratory parameters between the control group and KD group were analyzed. The levels of $\mathrm{L} \%, \mathrm{PA}$ and $\mathrm{CD}^{+}, \mathrm{CD} 4^{+} \mathrm{T}$ cell were lower in KD individuals. Higher levels of WBC, N\%, CRP, ESR, NTproBNP, ALT and $C D 4^{+} / C D 8^{+}$in $\mathrm{KD}$ group. these differences were statistically significant $(\mathrm{P}<0.05$ or $P<0.01)$. There were no other statistically significant differences in indicators of $P C T, T B I L, A L B$ and AST between the KD patients and control group (Table 2, Figure 1).

\section{Demographic data}

The demographic and clinical characteristics of the study participants are shown in Table 3. The acute infections among the normal control group were upper or lower respiratory tract infections or gastroenteritis. Of all the participants, 32 patients (25.40\%) had CAL formation, and 53 patients (42.06\%) were IVIG-resistant.

\section{Detecting HMGB1 and S100A12 expression}

Comparison with KD group, the relative expression levels of HMGB1 and S100A12 mRNA were higher than NC group $(P<0.05$, Figure3A). Subsequentely, compared with IVIG-responsive group, the mRNA levels of HMGB1 and S100A12 in IVIG-resistant group were markedly elevated $(P<0.05$, Figure3B). Moreover, the Real-time PCR melt curve indicated that the primers of S100A12 and HMGB1 were specific (Figure3C-D).

\section{Serum IL-17A measurements by ELISA}

IL-17A expression levels increased significantly in KD group $(P<0.01$, Figure4A). Also, the serum level of IL-17A in IVIG-resistant group was higher than IVIG-responsive group $(P<0.01$, Figure4B).

\section{Laboratory characteristics in IVIG-responsive and IVIG-resistant group}

In the KD group, the laboratory data of the IVIG-responsive patients and the IVIG-resistant patients were obtained before IVIG-treatment. The variance of laboratory parameters between the IVIG-responsive and the IVIG-resistant were analyzed. In terms of laboratory data of KD group, patients who were IVIGresistant had higher levels of N\%, CRP, NT-proBNP, TBIL, ALT and AST prior to IVIG therapy when compared with those who were IVIG-responsive. Meanwhile, compared with IVIG-responsive patients, the IVIG-resistant patients had lower levels both of L\% and PLT. PCT, ESR, ALB, CD $4^{+}, \mathrm{CD} 8^{+}$and $\mathrm{CD} 4^{+} / \mathrm{CD} 8^{+}$ expression levels remained higher than normal value. However, there had no significant difference when compared with IVIG-responsive patients and the IVIG-resistant patients (Table 4, Figure 2).

\section{Improvement of clinical symptoms in the IVIG-resistant after different treatments}


The recovery of clinical symptoms in the acute phase of the three groups of IVIG resistance after discharge were shown in Table 5. The study compared the effects of three adjunctive therapies from seven aspects: the pyrexia before starting treatment, average time of hospital stay, antipyretic time, mucosal congestion subsiding time, lymph node swelling subsiding time, redness and swelling of the hands and feet subsiding time and CALs formation before starting treatment. Among 53 IVIG resistance patients, 16 of them had CAL formation before treatment. The antipyretic time and mucosal congestion/redness and swelling of the hands and feet subsiding time of methylprednisolone group and infliximab group were all shorter than IVIG retreament group $(P<0.05$ or $P<0.01)$. There had no difference between three treatment options in lymph node swelling subsiding time $(P>0.05)$. All the follow up information was collected within 2 weeks and 2 months after discharge respectively.

\section{Various laboratory characteristics in the IVIG-resistant after different treatments}

Patients who were IVIG-resistant had no statistical difference in the following laboratory data before KD1 and KD2 when different drugs treatment during hospitalization. In KD3, compared with IVIG retreatment and infliximab group, methylprednisolone group showed more significant lowering CRP $(P<0.05)$. The other laboratory characteristics after three adjunctive therapies, there had no significantly difference $(P>0.05$, Table 6$)$. After discharge, we found that methylprednisolone group and infliximab group were more effective than IVIG retreatment group in alleviating inflammatory indexes $(P<0.05$ or $P<0.01$, Table 5). All the follow up information was collected within 2 weeks and 2 months after discharge respectively.

\section{Discussion}

Kawasaki disease (KD) is an acute febrile illness associated with vasculitis that affects infants and young children. It has become the main cause of acquired heart disease during childhood. And the coronary artery lesion significantly impair the quality of life [28-30]. The coronary wall inflammation, endothelial dysfunction and impaired vascular remodeling contribute to the development of coronary artery abnormalities (CAAs) and thrombosis[31]. Developing the early judgment of the factors of IVIGresistant and CALs is crucial. Although several predictive biomarkers and genes have been described, follow-up studies are inadequate. Especially consistent reported for the risk factors of IVIG-resistant and developing CALs. The precise mechanisms of IVIG-resistant and anti-inflammation in KD patients still remain unclear[13]. Our study aimed to identify the predictor for IVIG resistance, we investigated laboratory data collected before the initial IVIG treatment. IVIG resistant patients have higher levels of N\%, CRP, NT-proBNP, TBIL, ALT, AST and lower levels of L\%, PLT. Several previous studies reported that CRP, ESR, ALT, Y-GT and NT- proBNP could be used for predicting resistance to IVIG therapy and patients who are at high risk for CALs[4, 31-34]. To predict the ability of response to IVIG before initial therapy, would allow clinicians to identify those potential IVIG resistant patients and give them more aggressive treatments[35]. Our results demonstrated that the higher levels of WBC, N\%, CRP, ESR, NT-proBNP, ALT, $\mathrm{CD} 4^{+} / \mathrm{CD} 8^{+}$ratio and lower levels of $\mathrm{L} \%, \mathrm{PA}, \mathrm{CD} 8^{+}, \mathrm{CD} 4^{+}$can be considered as predictor for IVIG-resistant. 
Our data suggest that resistant to initial IVIG treatment and are a high risk for CALs may due to severe coronary inflammation.

The DAMPs such as HMGB1 and S100A12 have proven to be sensitive markers for disease activity and inflammation in numerous inflammatory disorders[36-38]. KD triggers the release of HMGB1 and S100A12, which activate toll-like receptors (TLRs) and receptor for advanced glycation endproducts (RAGE) in the affected area, leading to an exaggerated inflammatory response and cell death[39]. Here, we found significant increased levels of HMGB1 and S100A12 in total KD patients, especially in IVIGresistance group. These results indicating that HMGB1 and S100A12 maybe can help to assess the severity of vasculitis in KD. Th17 cells are a newly discovered T-helper cell subset associated with the proinflammatory stage of autoimmunity. Their differentiation requires both TGF-b combined with either IL-10 or IL-21 and results in the specific expression transcription factor retinoic acid-related orphan receptor ct(RORct), and the production of inflammatory cytokines including IL-6 and IL-17A[40]. Th17- and Tregrelated Cytokines participats in many autoimmune diseases. Among them, IL-17A is obviously associated with $K D$ and to provoke proinflammatory responses[13, 41]. In addition, studies reported that genetic aberrations in certain intracellular signaling pathways involving immune effector functions can increased susceptibility to KD and development of CALs[31]. In our study, we found the level of IL-17A increased in KD group before the initial treatment. Consistent with this, IL-17A remains at a high level with IVIGresistant patients. In addition, KD group had lower percentage of $\mathrm{CD} 4^{+} \mathrm{T}$ cell, $\mathrm{CD} 8^{+} \mathrm{T}$ cells and higher $\mathrm{CD} 4^{+} / \mathrm{CD} 8^{+}$ratio when compared to the NC group. Clinical risk factors predict patient resistance to IVIG and treatment regimens to reduce the risk of developing CALs which remain controversy. These findings strongly suggest that compared with the IVIG responsive group, the immune disorder may be more significant and serious in the IVIG resistant group.

Although IVIG is a powerful anti-inflammatory and immune regulating drug, there are still some children can't relieve the clinical symptoms after initial IVIG treatment effectively. IVIG resistance as one of the most important issue to be solved urgently for KD patients. Many experts recommend retreatment with a second dose of IVIG for IVIG-resistant. But in our study, IVIG retreatment did not show a particular advantage in alleviating clinical symptoms and inflammatory indicators. Therefore, how to choose proper therapies for IVIG-resistant KD should based on well understanding of the mechanism of that resistance. Results from our laboratory suggests that the striking anti-inflammatory effects of IVIG were weakened by addition of excessive activate inflammatory response. For IVIG-resistant, we show the IVIG retreatment, methylprednisolone, and infliximab which have different advantages in suppression of the inflammation. Compared with infliximab and IVIG retreatment group, the methylprednisolone showed more advantages in reducing $C R P$ in the short-term follow-up $(P<0.05)$. But there's no difference between the three treatments in alleviate the levels of WBC, N \%, ESR, ALB, ALT, AST and PLT $(P>0.05)$. Although whether to the use of corticosteroids in KD is still controversial, in our study methylprednisolone group showed better in improving clinical symptoms than the IVIG retreatment and infliximab group during hospitalization. After discharge, we found that methylprednisolone group and infliximab group were more effective than IVIG retreatment group in alleviating inflammatory indexes at follow-up. The main Complications of 
infliximab is that it can increase the risk of latent infection or opportunistic infection. Fortunately, there's no infusion reactions or complications were happened in our study. We suggests that infliximab is effective and well tolerated for IVIG-resistant KD. It can also possible to prevent CALs effectively by inhibiting overactive inflammatory response.

In our study, several details deserve further attention. First, the relatively small sample size of this study might prevent some of the detected data from being statistically significant. Second, our study results need to be validated across different hospitals, different regions, different races. Lastly, IVIG-resistant was associated with higher levels of HMGB1and S100A12, but the exact signaling pathway remains unknown. Therefore, further work to explore the mechanism of IVIG-resistant is necessary. Further studies on the mechanism of IVIG-resistant may help identify new methods to predict and treat IVIG-resistant patients effectively in KD.

\section{Conclusions}

Accumulating evidence suggests that higher levels of WBC, N\%, CRP, ESR, NT-proBNP, ALT, CD4 $4^{+} / \mathrm{CD} 8^{+}$ ratio and lower levels of $\mathrm{L} \%, \mathrm{PA}, \mathrm{CD} 8^{+}, \mathrm{CD} 4^{+} \mathrm{T}$ cells showed useful for predicting in KD patients with IVIGresistant. And high level of HMGB1, S100A12 and serum IL-17A before the initial treatment could also predict IVIG-resistant. In addition, methylprednisolone showed better outcome in improving clinical symptoms than the IVIG retreatment and infliximab.

\section{Abbreviations}

KD Kawasaki disease

IVIG Intravenous immunoglobulin

CALs coronary artery lesions

DAMPs damage-associated molecular pattern molecules

HMGB1 high-mobility group protein B1

S100A12 S100 calcium-binding protein A12

IL-17A Interleukin-17A

\section{Declarations}

\section{Ethics approval and consent to participate}

Written informed consents were obtained from parents or guardians of all study participants. The experimental protocol was approved by the Ethics Committee of Wuhan Children's Hospital. 


\section{Consent for publication}

All the authors agreed to publish.

\section{Availability of data and material}

The data used and analyzed in the present study are available from the corresponding author on reasonable request.

\section{Competing interests}

The authors declare that they have no competing interest.

\section{Funding}

The study was funded by Hubei Province Health and Family Planning Commission of Scientific Research Projec (Nos. WJ2017M197 and WJ2019H313), Wuhan City Health and Family Planning Commission of clinical medical research major project (No.WX19M03)

\section{Authors' contributions}

DY and PL carried out the experiments, and analyzed, and interpreted the data. FL and WY were responsible for the collection of blood samples and the interpretation of the data from the clinical perspective. DY contributed to the conception and design of the study, the analysis and interpretation of the data, and drafting and revising the manuscript. All authors read and approved the final manuscript.

\section{Acknowledgements}

We sincerely thank the patients for their participation in this study, as well as the Key Laboratory of Wuhan Children's hospital.

\section{References}

1. McCrindle BW, Rowley AH, Newburger JW, Burns JC, Bolger AF, Gewitz M, et al. Diagnosis, Treatment, and Long-Term Management of Kawasaki Disease: A Scientific Statement for Health Professionals From the American Heart Association. CIRCULATION. 2017;135:e927-99.

2. Lee HY, Song MS. Predictive factors of resistance to intravenous immunoglobulin and coronary artery lesions in Kawasaki disease. Korean J Pediatr. 2016;59:477-82.

3. Ou-Yang MC, Kuo HC, Lin IC, Sheen JM, Huang FC, Chen CC, et al. Plasma clusterin concentrations may predict resistance to intravenous immunoglobulin in patients with Kawasaki disease. ScientificWorldJournal. 2013; 2013:382523.

4. Baek JY, Song MS. Meta-analysis of factors predicting resistance to intravenous immunoglobulin treatment in patients with Kawasaki disease. Korean J Pediatr. 2016;59:80-90. 
5. Giulia A, Emely V, Carolin P, et al. Monocyte-Derived Interleukin-1 $\beta$ As the Driver of S100A12-Induced Sterile Inflammatory Activation of Human Coronary Artery Endothelial Cells: Implications for the Pathogenesis of Kawasaki Disease. Arthritis Rheumatol, 2019;71(5):792-804.

6. Kuo H, Wong HS, Chang W, Chen B, Wu M, Yang KD, et al. Prediction for Intravenous Immunoglobulin Resistance by Using Weighted Genetic Risk Score Identified From Genome-Wide Association Study in Kawasaki DiseaseCLINICAL PERSPECTIVE. Circulation: Cardiovascular Genetics. 2017; 10:e1625.

7. Agarwal S, Agrawal DK. Kawasaki disease: etiopathogenesis and novel treatment strategies. EXPERT REV CLIN IMMU. 2017;13:247-58.

8. Shin J, Lee H, Eun L. Verification of Current Risk Scores for Kawasaki Disease in Korean Children. J KOREAN MED SCI. 2017; 32:1991.

9. Qi Y, Gong F, Zhang Q, Xie C, Wang W, Fu S. Reverse regulation of soluble receptor for advanced glycation end products and proinflammatory factor resistin and S100A12 in Kawasaki disease. ARTHRITIS RES THER. 2012;14:R251.

10. Jong G, Yoonsun B, Dongjik S, et al. HMGB1 Gene Polymorphism Is Associated With Coronary Artery Lesions and Intravenous Immunoglobulin Resistance in Kawasaki Disease. Rheumatology. 2019;58(5):770-5.

11. Galeotti C, Kaveri SV, Bayry J. Molecular and immunological biomarkers to predict IVIg response. TRENDS MOL MED. 2015;21:145-7.

12. Rajni K, Pandiarajan V, Amit R, et al. Immunogenetics of Kawasaki disease. Clin Rev Allergy Immunol. 2020; 5(21).

13. Guo MM, Tseng WN, Ko CH, Pan HM, Hsieh KS, Kuo HC. Th17- and Treg-related cytokine and mRNA expression are associated with acute and resolving Kawasaki disease. ALLERGY. 2015;70:310-8.

14. Jia S, Li C, Wang G, Yang J, Zu Y. The T helper type 17/regulatory T cell imbalance in patients with acute Kawasaki disease. Clinical Experimental Immunology. 2010;162:131-7.

15. Clark DE, Denby KJ, Kaufman LM, Fill MA, Piya B, Krishnaswami S, et al. Predictors of Intravenous Immunoglobulin Non-Response and Racial Disparities in Kawasaki Disease. The Pediatric Infectious Disease Journal. 2018:1.

16. Inamo Y, Saito K, Hasegawa M, Hayashi R, Nakamura T, Abe O, et al. Effect of dalteparin, a lowmolecular-weight heparin, as adjunctive therapy in patients with Kawasaki disease: a retrospective study. BMC PEDIATR. 2014;14:27.

17. Takeuchi M, Inuzuka R, Hayashi T, Shindo T, Hirata Y, Shimizu N, et al. Novel Risk Assessment Tool for Immunoglobulin Resistance in Kawasaki Disease: Application Using a Random Forest Classifier. PEDIATR INFECT DIS J. 2017;36:821-6.

18. Sano T, Kurotobi S, Matsuzaki K, Yamamoto T, Maki I, Miki K, et al. Prediction of non-responsiveness to standard high-dose gamma-globulin therapy in patients with acute Kawasaki disease before starting initial treatment. EUR J PEDIATR. 2007;166:131-7.

19. Egami K, Muta H, Ishii M, Suda K, Sugahara Y, lemura M, et al. Prediction of resistance to intravenous immunoglobulin treatment in patients with Kawasaki disease. J Pediatr. 2006;149:237-40. 
20. Kim MK, Song MS, Kim GB. Factors Predicting Resistance to Intravenous Immunoglobulin Treatment and Coronary Artery Lesion in Patients with Kawasaki Disease: Analysis of the Korean Nationwide Multicenter Survey from 2012 to 2014. KOREAN CIRC J. 2018;48:71.

21. Tremoulet AH, Best BM, Song S, Wang S, Corinaldesi E, Eichenfield JR, et al. Resistance to intravenous immunoglobulin in children with Kawasaki disease. J Pediatr. 2008;153:117-21.

22. Tremoulet AH, Best BM, Song S, Wang S, Corinaldesi E, Eichenfield JR, et al. Resistance to Intravenous Immunoglobulin in Children with Kawasaki Disease. The Journal of Pediatrics. 2008;153:117-21.

23. Tremoulet AH, Jain S, Jaggi P, Jimenez-Fernandez S, Pancheri JM, Sun $X$, et al. Infliximab for intensification of primary therapy for Kawasaki disease: a phase 3 randomised, double-blind, placebo-controlled trial. LANCET. 2014;383:1731-8.

24. Shulman ST, Rowley AH. Kawasaki disease: insights into pathogenesis and approaches to treatment. NAT REV RHEUMATOL. 2015;11:475-82.

25. Burns JC, Franco A. The immunomodulatory effects of intravenous immunoglobulin therapy in Kawasaki disease. Expert Rev Clin Immunol. 2015;11:819-25.

26. Burns JC, Best BM, Mejias A, Mahony L, Fixler DE, Jafri HS, et al. Infliximab Treatment of Intravenous Immunoglobulin-Resistant Kawasaki Disease. The Journal of Pediatrics. 2008;153:833-8.

27. Ding Y, Liu P, Chen Z, Zhang S, Wang Y, Cai X, et al. Emodin Attenuates Lipopolysaccharide-Induced Acute Liver Injury via Inhibiting the TLR4 Signaling Pathway in vitro and in vivo. FRONT PHARMACOL. 2018; 9.

28. Kuo HC, Liang CD, Wang CL, Yu HR, Hwang KP, Yang KD. Serum albumin level predicts initial intravenous immunoglobulin treatment failure in Kawasaki disease. ACTA PAEDIATR. 2010;99:1578-83.

29. Wakiguchi H, Hasegawa S, Suzuki Y, Kudo K, Ichiyama T. Relationship between T-cell HLA-DR expression and intravenous immunoglobulin treatment response in Kawasaki disease. PEDIATR RES. 2015;77:536-40.

30. Abe J, Jibiki T, Noma S, Nakajima T, Saito H, Terai M. Gene expression profiling of the effect of highdose intravenous Ig in patients with Kawasaki disease. J IMMUNOL. 2005;174:5837-45.

31. Rajni K, Pandiarajan V, Amit R, et al. Immunogenetics of Kawasaki disease. Review Clin Rev Allergy Immunol. 2020;5:3.

32. Sittiwangkul R, Pongprot Y, Silvilairat S, Phornphutkul C. Management and outcome of intravenous gammaglobulin-resistant Kawasaki disease. Singapore Med J. 2006;47:780-4.

33. Wang Y, Li Z, Hu G, Hao S, Deng X, Huang M, et al. Unique Molecular Patterns Uncovered in Kawasaki Disease Patients with Elevated Serum Gamma Glutamyl Transferase Levels: Implications for Intravenous Immunoglobulin Responsiveness. PLOS ONE. 2016;11:e167434.

34. Sileikiene R, Kudzyte J, Jankauskas A, Labanauskas L, Rakauskiene V, Jurkiene N, et al. Rare refractory Kawasaki disease in an adolescent boy with cardiac and diffuse coronary artery involvement. Medicina. 2013;49:341-5. 
35. Lee HY, Song MS. Predictive factors of resistance to intravenous immunoglobulin and coronary artery lesions in Kawasaki disease. Korean Journal of Pediatrics. 2016;59:477.

36. Fu S, Gong F, Xie C, Zhu W, Wang W, Shen H, et al. S100A12 on circulating endothelial cells surface in children with Kawasaki disease. PEDIATR RES. 2010;68:165-8.

37. Fury W, Tremoulet AH, Watson VE, Best BM, Shimizu C, Hamilton J, et al. Transcript abundance patterns in Kawasaki disease patients with intravenous immunoglobulin resistance. HUM IMMUNOL. 2010;71:865-73.

38. Foell D, Ichida F, Vogl T, Yu X, Chen R, Miyawaki T, et al. S100A12 (EN-RAGE) in monitoring Kawasaki disease. LANCET. 2003;361:1270-2.

39. Lok KZ, Basta M, Manzanero S, Arumugam TV. Intravenous immunoglobulin (IVIg) dampens neuronal toll-like receptor-mediated responses in ischemia. J Neuroinflammation. 2015;12:73.

40. Wu S, Zhang X, Zhou N. [Up-regulation of serum- and glucocorticoid-inducible kinase 1 (SGK1) of CD4(+) T cells is positively related to RORC and IL-17A in patients with Kawasaki disease]. Xi Bao Yu Fen Zi Mian Yi Xue Za Zhi. 2015;31:1378-82.

41. Jia S, Li C, Wang G, Yang J, Zu Y. The T helper type 17/regulatory T cell imbalance in patients with acute Kawasaki disease. CLIN EXP IMMUNOL. 2010;162:131-7.

\section{Tables}

Table 1

The sequences of all primers for it-PCR.

\begin{tabular}{|c|c|c|}
\hline Gene & primer sequence & Human $\left(5^{\prime} \rightarrow 3^{\prime}\right)$ \\
\hline \multirow[t]{2}{*}{ HMGB1 } & forward primer & 5'-TCAAAGGAGAACATCCTGGCCTGT-3' \\
\hline & reverse primer & 5'-CTGCTTGTCATCTGCAGCAGTGTT-3' \\
\hline \multirow[t]{2}{*}{ S100A12 } & forward primer & 5'-TCTAAGGGTGAGCTGAAGCAG-3' \\
\hline & reverse primer & 5'-CAATGGCTACCAGGGATATGA-3' \\
\hline \multirow[t]{2}{*}{ GAPDH } & forward primer & 5'-GGTGAAGGTCGGAGTCAACGG-3' \\
\hline & reverse primer & 5'-GGTCATGAGTCCTTCCACGATACC-3' \\
\hline
\end{tabular}


Table 2

Laboratory characteristics of the KD group and control group

\begin{tabular}{|lll|}
\hline Parameter & Control $(\mathrm{N}=16)$ & $\mathrm{KD}(\mathrm{N}=126)$ \\
\hline WBC & $11.32 \pm 1.24$ & $15.15 \pm 3.32^{\star}$ \\
\hline Neutrophil(\%) & $56.74 \pm 6.32$ & $68.08 \pm 1.50^{\star}$ \\
\hline Lymphocyte(\%) & $34.66 \pm 6.08$ & $24.57 \pm 1.46^{*}$ \\
\hline PLT & $268.10 \pm 19.28$ & $362.30 \pm 12.85^{*}$ \\
\hline CRP & $33.28 \pm 10.73$ & $95.50 \pm 5.54^{* *}$ \\
\hline PCT & $0.78 \pm 0.34$ & $3.02 \pm 0.86$ \\
\hline ESR & $24.13 \pm 4.84$ & $47.67 \pm 2.37 *$ \\
\hline NT-proBNP & $316.70 \pm 32.61$ & $1042.00 \pm 129.80^{*}$ \\
\hline Total bilirubin & $9.31 \pm 1.02$ & $18.15 \pm 2.00$ \\
\hline Albumin & $42.34 \pm 0.82$ & $38.34 \pm 2.50$ \\
\hline ALT & $17.38 \pm 3.00$ & $63.87 \pm 7.19 *$ \\
\hline AST & $36.00 \pm 3.64$ & $64.63 \pm 13.35$ \\
\hline PA & $114.50 \pm 10.04$ & $75.36 \pm 4.36 *$ \\
\hline CD8+ & $27.12 \pm 3.94$ & $18.58 \pm 0.59^{* *}$ \\
\hline CD4+ & $38.97 \pm 2.31$ & $33.42 \pm 0.88^{*}$ \\
\hline CD4+/CD8+ & $1.47 \pm 0.20$ & $2.03 \pm 0.08^{*}$ \\
\hline *P<0.05, **P<0.01 KD group VS control group. \\
\hline
\end{tabular}

Table 3

Demographic and clinical characteristics of total KD patients

\begin{tabular}{|ll|}
\hline characteristics & Patients with $\mathrm{KD}(\mathbf{N}=\mathbf{1 2 6})$ \\
\hline Median age range (years) & $1.85 \pm 0.20$ \\
\hline Male $(\mathrm{n}(\%))$ & 65.87 \\
\hline Pyrexia & $5.81 \pm 0.26$ \\
\hline CAL formation & 32 \\
\hline IVIG resistant & 53 \\
\hline
\end{tabular}


Table 4

Laboratory characteristics in IVIG-responsive and IVIG-resistant group

\begin{tabular}{|c|c|c|}
\hline Parameter & IVIG-responsive $(\mathrm{N}=73$ ) & $\begin{array}{l}\text { IVIG-resistant } \\
(\mathrm{N}=53)\end{array}$ \\
\hline WBC & $14.39 \pm 0.6674$ & $16.19 \pm 0.9670$ \\
\hline Neutrophil(\%) & $61.30 \pm 2.112$ & $73.63 \pm 2.226^{\star \star}$ \\
\hline Lymphocyte(\%) & $28.71 \pm 1.820$ & $18.87 \pm 2.174^{\star \star}$ \\
\hline PLT & $391.4 \pm 16.07$ & $322.3 \pm 19.94$ *夫 \\
\hline CRP & $84.55 \pm 7.412$ & $110.6 \pm 7.957 *$ \\
\hline РCT & $2.441 \pm 1.378$ & $3.825 \pm 0.7427$ \\
\hline ESR & $44.32 \pm 2.913$ & $52.30 \pm 3.901$ \\
\hline NT-proBNP & $614.4 \pm 58.35$ & $1746 \pm 315.3^{\star \star}$ \\
\hline Total bilirubin & $13.77 \pm 2.248$ & $24.19 \pm 3.458^{\star \star}$ \\
\hline Albumin & $37.54 \pm 0.5155$ & $39.45 \pm 5.917$ \\
\hline ALT & $47.23 \pm 6.292$ & $86.77 \pm 14.24$ ** \\
\hline AST & $41.45 \pm 3.543$ & $96.57 \pm 30.99 *$ \\
\hline PA & $76.54 \pm 4.301$ & $73.73 \pm 8.569$ \\
\hline $\mathrm{CD}^{+}$ & $19.12 \pm 0.7727$ & $17.83 \pm 0.9165$ \\
\hline $\mathrm{CD}^{+}$ & $35.07 \pm 1.261$ & $33.04 \pm 1.408$ \\
\hline $\mathrm{CD}^{+} / \mathrm{CD}^{+}$ & $2.030 \pm 0.1050$ & $2.037 \pm 0.1106$ \\
\hline $\begin{array}{l}\text { General analysi } \\
\text { patients. }{ }^{*} P<0 \text {. }\end{array}$ & $\mathrm{d}$ isolated from KD patie & sponsive and IVIG-resistant \\
\hline
\end{tabular}


Table 5

The recovery of clinical symptoms after different adjunctive therapies. ${ }^{*} P<0.05,{ }^{*} \mathrm{P}<0.01$ compared with IVIG retreatment group; \#P< 0.05 Methylprednisolone group VS Infliximab group. A: index recovery within two weeks after discharge; B: index recovery within two months after discharge.

\begin{tabular}{|c|c|c|c|}
\hline Item & $\begin{array}{l}\text { IVIG } \\
\text { retreatment(N = } \\
19)\end{array}$ & $\begin{array}{l}\text { methylprednisolone( } \mathrm{N} \\
=21)\end{array}$ & $\begin{array}{l}\text { infliximab }(\mathrm{N} \\
=13)\end{array}$ \\
\hline Pyrexia before starting treatment & $6.17 \pm 1.82$ & $5.94 \pm 1.71$ & $6.02 \pm 1.94$ \\
\hline hospital stay & $9.11 \pm 2.41$ & $8.23 \pm 2.23$ & $8.69 \pm 2.62$ \\
\hline antipyretic time & $1.21 \pm 0.80$ & $0.75 \pm 0.51^{*}$ & $0.70 \pm 0.46^{*}$ \\
\hline Mucosal congestion subsiding time & $1.19 \pm 0.47$ & $0.82 \pm 0.39 * \star$ & $0.83 \pm 0.35^{\star}$ \\
\hline Lymph node swelling subsiding time & $3.47 \pm 1.36$ & $2.99 \pm 1.32$ & $3.24 \pm 1.46$ \\
\hline $\begin{array}{l}\text { redness and swelling of the hands and } \\
\text { feet subsiding time }\end{array}$ & $1.98 \pm 0.56$ & $1.47 \pm 0.61^{\star \star}$ & $\begin{array}{l}1.35 \pm \\
0.67 \star \star\end{array}$ \\
\hline $\begin{array}{l}\text { CAL formation before starting } \\
\text { treatment }\end{array}$ & $6 / 19$ & $6 / 21$ & $4 / 13$ \\
\hline \multicolumn{4}{|l|}{$\begin{array}{l}\text { Follow-up after discharge of index } \\
\text { recovery }\end{array}$} \\
\hline \multicolumn{4}{|l|}{ (A:two weeks; B:two months) } \\
\hline $\mathrm{A}(\mathrm{CAL}$ recovery $)$ & $0 / 6$ & $1 / 6$ & $1 / 4$ \\
\hline $\mathrm{A}(\mathrm{WBC})$ & $7.71 \pm 0.58$ & $7.52 \pm 0.64$ & $8.76 \pm 1.74$ \\
\hline A (Neutrophil(\%)) & $65.15 \pm 2.02$ & $60.65 \pm 2.29 * \#$ & $\begin{array}{l}62.36 \pm \\
2.03^{*}\end{array}$ \\
\hline $\mathrm{A}(\mathrm{PLT})$ & $703.15 \pm 56.38$ & $701.57 \pm 59.87 \#$ & $\begin{array}{l}789.20 \pm \\
72.35^{\star \star}\end{array}$ \\
\hline $\mathrm{A}(\mathrm{CRP})$ & $13.63 \pm 2.36$ & $11.47 \pm 3.02^{\star}$ & $\begin{array}{l}10.36 \pm \\
2.87^{\star \star}\end{array}$ \\
\hline $\mathrm{B}(\mathrm{CAL}$ recovery $)$ & $1 / 6$ & $2 / 6$ & $1 / 4$ \\
\hline $\mathrm{B}(\mathrm{WBC})$ & $5.98 \pm 0.38$ & $6.57 \pm 0.69 * \star$ & $\begin{array}{l}6.84 \pm \\
1.02^{\star \star}\end{array}$ \\
\hline B (Neutrophil(\%)) & $57.36 \pm 2.78$ & $59.15 \pm 1.69 *$ & $\begin{array}{l}54.10 \pm \\
3.44^{\star \star \#}\end{array}$ \\
\hline $\mathrm{B}(\mathrm{PLT})$ & $322.11 \pm 23.68$ & $299.87 \pm 30.68^{*}$ & $\begin{array}{l}311.52 \pm \\
34.55\end{array}$ \\
\hline $\mathrm{B}(\mathrm{CRP})$ & $7.22 \pm 1.98$ & $6.25 \pm 2.54 \#$ & $\begin{array}{l}3.68 \pm \\
2.39 \star \star\end{array}$ \\
\hline
\end{tabular}


Table 6

Various laboratory characteristics in the IVIG-resistant after different treatments

\section{IVIG-resistant $(\mathrm{N}=53)$}

\begin{tabular}{|c|c|c|c|c|}
\hline & Parameter & IVIG retreatment(19) & Methylprednisolone(21) & Infliximab(13) \\
\hline & WBC & $17.03 \pm 2.04$ & $14.79 \pm 1.30$ & $14.16 \pm 1.60$ \\
\hline \multirow[t]{8}{*}{ KD1 } & Neutrophil(\%) & $71.09 \pm 4.70$ & $78.33 \pm 2.62$ & $78.48 \pm 2.30$ \\
\hline & PLT & $357.30 \pm 38.87$ & $297.40 \pm 19.70$ & $315.50 \pm 31.43$ \\
\hline & CRP & $97.43 \pm 10.08$ & $133.00 \pm 28.93$ & $132.80 \pm 15.02$ \\
\hline & ESR & $51.47 \pm 6.79$ & $54.43 \pm 6.71$ & $56.15 \pm 7.80$ \\
\hline & ALB & $52.68 \pm 16.27$ & $34.30 \pm 1.35$ & $33.98 \pm 1.74$ \\
\hline & ALT & $91.32 \pm 22.35$ & $112.6 \pm 26.56$ & $65.85 \pm 15.17$ \\
\hline & AST & $82.89 \pm 19.61$ & $92.33 \pm 19.24$ & $50.15 \pm 7.53$ \\
\hline & WBC & $14.68 \pm 1.22$ & $15.85 \pm 1.11$ & $18.57 \pm 1.30$ \\
\hline \multirow[t]{8}{*}{ KD2 } & Neutrophil(\%) & $56.44 \pm 4.14$ & $63.60 \pm 3.02$ & $68.03 \pm 4.05$ \\
\hline & PLT & $350.50 \pm 50.90$ & $399.40 \pm 44.83$ & $356.40 \pm 48.13$ \\
\hline & CRP & $75.22 \pm 12.13$ & $86.29 \pm 11.63$ & $104.10 \pm 16.82$ \\
\hline & ESR & $69.00 \pm 5.25$ & $77.52 \pm 5.43$ & $87.46 \pm 7.78$ \\
\hline & ALB & $28.26 \pm 0.84$ & $29.00 \pm 0.95$ & $27.80 \pm 0.90$ \\
\hline & ALT & $38.08 \pm 3.77$ & $41.48 \pm 6.54$ & $27.15 \pm 4.00$ \\
\hline & AST & $30.58 \pm 2.18$ & $30.05 \pm 3.64$ & $30.77 \pm 2.49$ \\
\hline & WBC & $15.85 \pm 1.41$ & $16.16 \pm 1.27$ & $15.99 \pm 1.82$ \\
\hline \multirow[t]{6}{*}{ KD3 } & Neutrophil(\%) & $59.92 \pm 4.67$ & $59.22 \pm 3.68$ & $54.55 \pm 4.94$ \\
\hline & PLT & $520.90 \pm 40.58$ & $633.20 \pm 52.49$ & $707.20 \pm 62.27$ \\
\hline & CRP & $50.63 \pm 17.80$ & $15.42 \pm 3.15^{\star}$ & $33.21 \pm 12.03$ \\
\hline & ESR & $76.16 \pm 5.03$ & $68.90 \pm 6.16$ & $77.62 \pm 7.93$ \\
\hline & ALB & $32.34 \pm 1.01$ & $30.95 \pm 1.48$ & $30.99 \pm 1.09$ \\
\hline & ALT & $32.81 \pm 8.45$ & $33.52 \pm 6.05$ & $19.31 \pm 2.45$ \\
\hline
\end{tabular}

Laboratory characteristics changes in IVIG-resistant patients during KD1, KD2 and KD3 points of Kawasaki disease. KD1: before the treatment of initial IVIG (A); KD2: after the treatment of initial IVIG (B); KD3: after the second dose of different treatment options, including IVIG retreatment, methylprednisolone and infliximab group (C). ${ }^{*} \mathrm{P}<0.05$ compared with IVIG-retreatment group. 


\begin{tabular}{|c|c|c|c|}
\hline \multicolumn{4}{|c|}{ IVIG-resistant(N = 53 ) } \\
\hline AST & $30.95 \pm 2.91$ & $32.38 \pm 4.35$ & $28.15 \pm 3.48$ \\
\hline \multicolumn{4}{|c|}{$\begin{array}{l}\text { Laboratory characteristics changes in IVIG-resistant patients during KD1, KD2 and KD3 points of } \\
\text { Kawasaki disease. KD1: before the treatment of initial IVIG (A); KD2: after the treatment of initial IVIG } \\
\text { (B); KD } \text { K: after the second dose of different treatment options, including IVIG retreatment, } \\
\text { methylprednisolone and infliximab group (C). }{ }^{*}<0.05 \text { compared with IVIG-retreatment group. }\end{array}$} \\
\hline
\end{tabular}

\section{Figures}

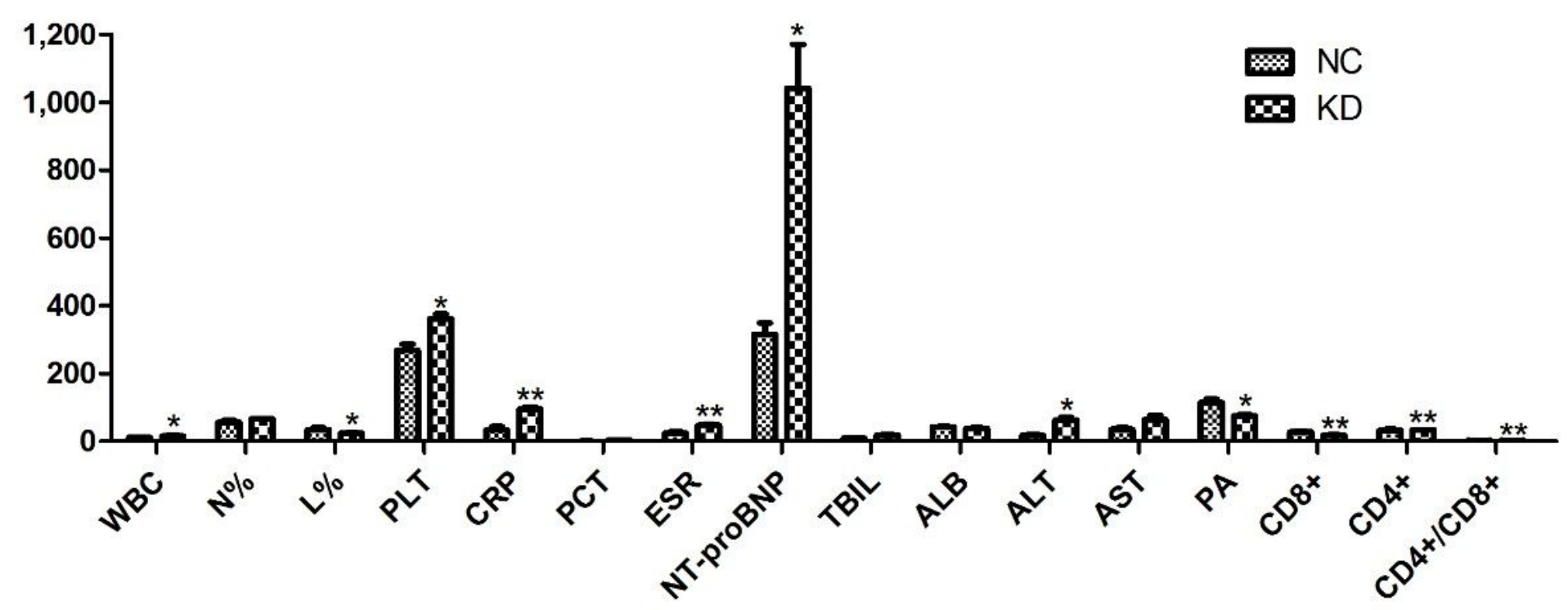

\section{Figure 1}

Laboratory characteristics between with KD group and NC group. NC group: normal control group; KD group: Kawasaki disease group. ${ }^{*} P<0.05,{ }^{* *} P<0.01$ 


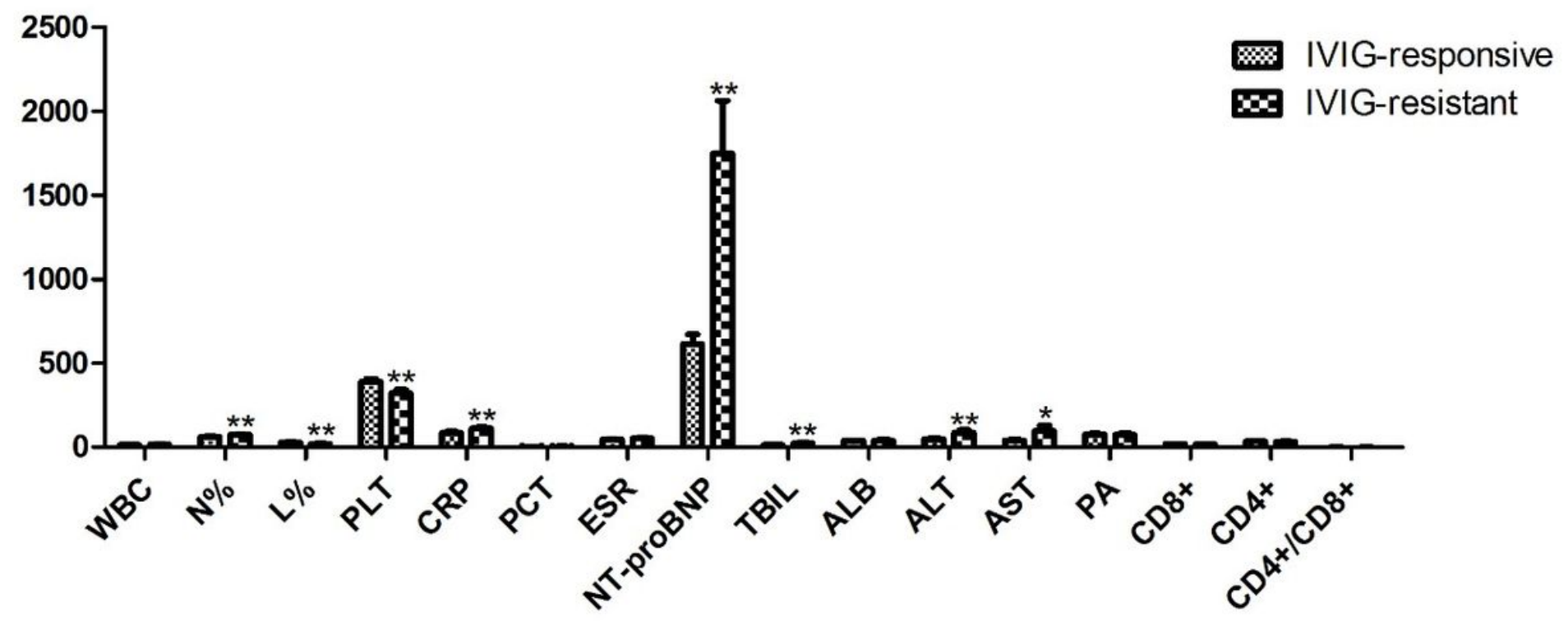

Figure 2

Laboratory characteristics between with IVIG-responsive and IVIG-resistant group ${ }^{*} P<0.05,{ }^{*} P<0.01$

A



C

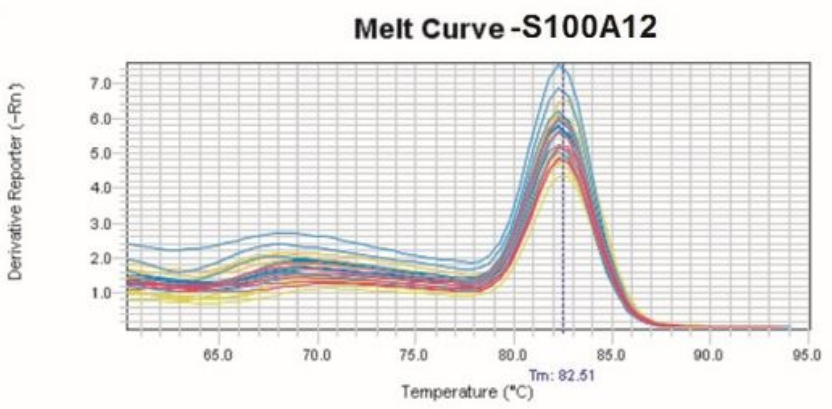

B



D

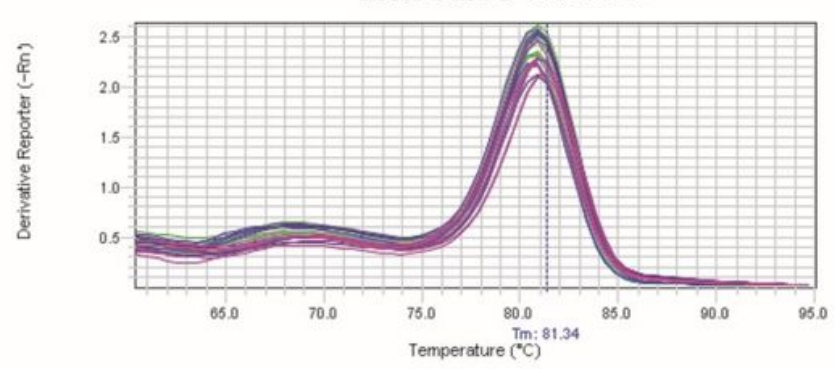

Figure 3 
(A): The relative expression levels of HMGB1 and S100A12 mRNA between NC group and KD group. (B) The relative expression levels of HMGB1 and S100A12 mRNA between IVIG-responsive group and IVIGresistant group. The melt curve of S100A12(C) and HMGB1(D).Data are shown as the mean \pm SD. \# : P< 0.05
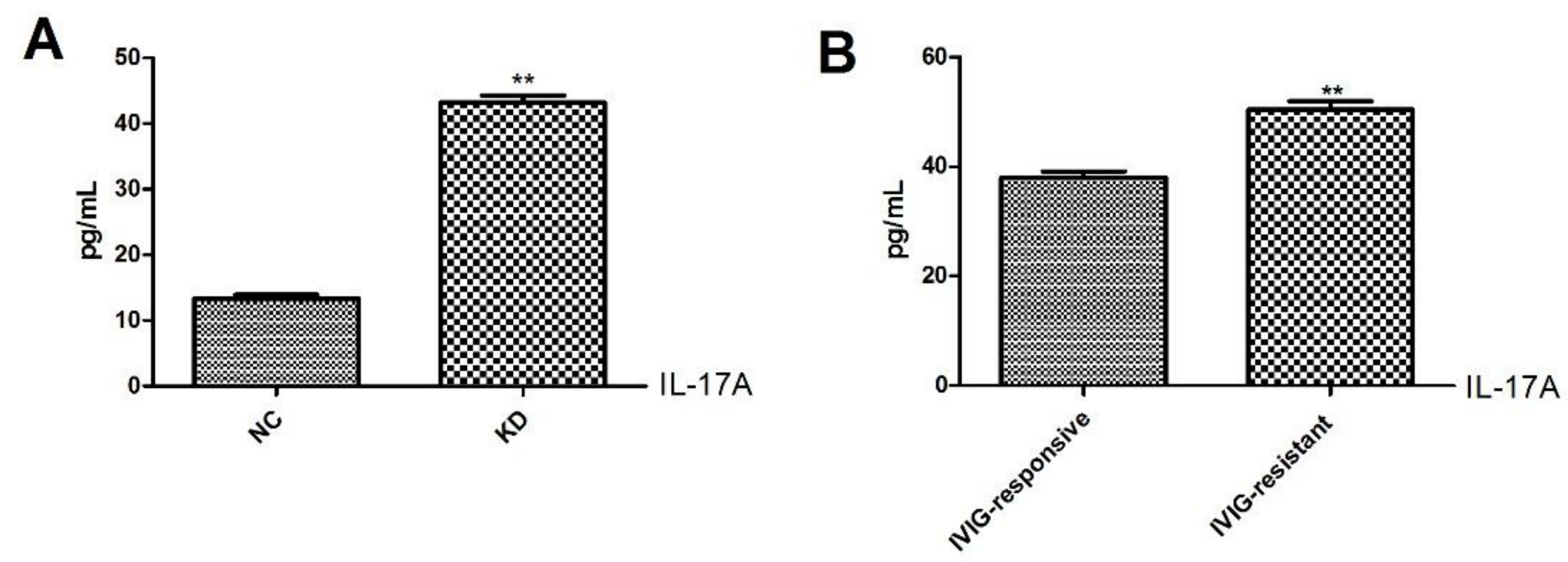

\section{Figure 4}

(A): The serum level of IL-17A measurements by ELISA between NC group and KD group. (B): The serum level of IL-17A measurements by ELISA between IVIG-responsive group VS IVIG-resistant group. Data are shown as the mean \pm SD. $* \star P<0.01$ 